\title{
A modified technique to overcome the limitations of intraductal ultrasound for diagnosis of common bile duct stones
}

The use of intraductal ultrasound at endoscopic retrograde cholangiopancreatography (ERCP) enables the identification of more than $95 \%$ of common bile duct stones, regardless of their size or composition, or the diameter of the duct [1]. However, false-positive studies have been reported to occur because of the inherent difficulty of differentiating common bile duct stones from air bubbles using intraductal ultrasound [1]. To overcome this limitation and achieve optimal imaging, we propose a new technique that can be adopted when intraductal ultrasound is performed at ERCP.

We prospectively evaluated ten patients who underwent intraductal ultrasound during ERCP for evaluation of "indeterminate" common bile duct filling defects. All the patients had undergone prior biliary sphincterotomy. These "indeterminate" common bile duct filling defects were defined as those defects identified in patients in whom an occlusion cholangiogram using a stone retrieval balloon had not been able to differentiate common bile duct stones from air bubbles. Intraductal ultrasound was performed using a 20-MHz ultrasound catheter probe (UM G20-29R; Olympus, Melville, New York, USA) connected to a standard endoscopic ultrasound processor (Olympus EU-M30). A 0.035-inch guide wire was first placed in the intrahepatic bile duct and the intraductal ultrasound catheter probe was advanced over the guide wire and slowly withdrawn in a stepwise fashion.

Intraductal ultrasound definitions were: an echo-rich focus in the bile duct, with or without acoustic shadowing, for common bile duct stones; comet-shaped echoes with acoustic shadowing or fanshaped echo-rich signals for air bubbles; and variably shaped echoes and easily distorted echo-rich structures without acoustic shadowing for sludge [1]. As the intraductal ultrasound image quality in our patients was poor because of inadequate acoustic coupling due to pneumobilia, an ERCP catheter was introduced

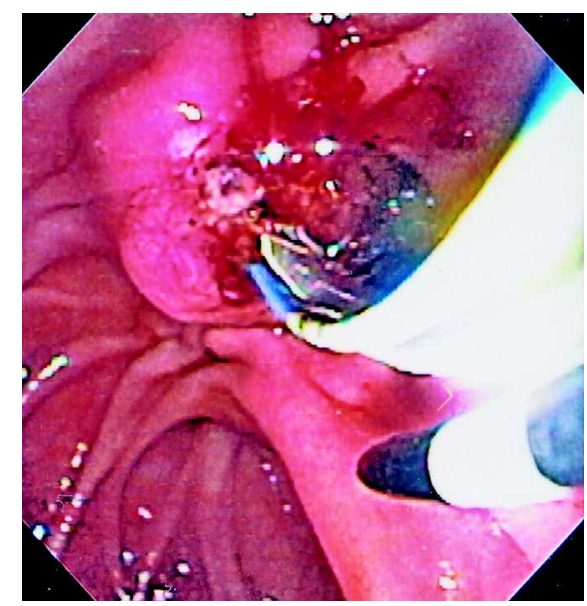

Figure 1 An endoscopic retrograde cholangiopancreatography catheter was introduced into the common bile duct, alongside the intraductal ultrasound probe, to flush normal saline into the common bile duct.

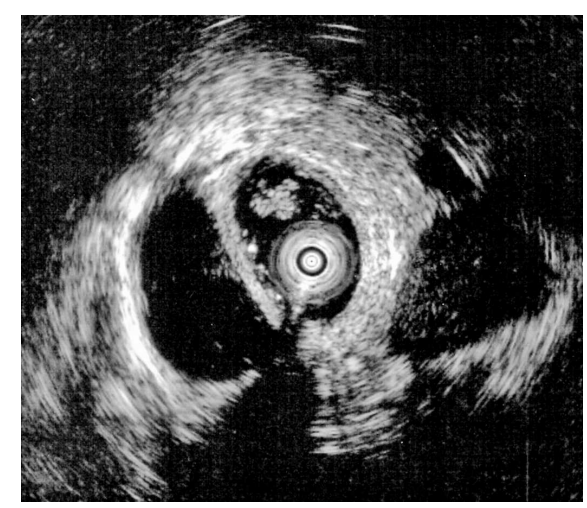

Figure 2 Better acoustic coupling after irrigation of normal saline into the common bile duct enabled visualization of biliary sludge that had not been seen previously.

into the common bile duct, alongside the intraductal ultrasound probe, and $25 \mathrm{ml}$ of normal saline was flushed into the common bile duct (Figure 1). The intraductal ultrasound probe was then gradually withdrawn. Better acoustic coupling enabled visualization of residual sludge/ stones in seven of the ten patients, which were extracted using a stone retrieval balloon/basket (Figure 2). In the three other patients the common bile duct appeared normal.
Flushing normal saline into the common bile duct during intraductal ultrasound examination improves acoustic coupling and enables reliable differentiation of common bile duct stones from air bubbles.

Endoscopy_UCTN_Code_TTT_1AS_2AD

\section{S. Varadarajulu}

Division of Gastroenterology-Hepatology, University of Alabama at Birmingham Medical Center, Birmingham, Alabama, USA.

\section{References}

${ }^{1}$ Tseng LJ, Jao YT, Mo LR et al. Over-the-wire US catheter probe as an adjunct to ERCP in the detection of choledocholithiasis. Gastrointest Endosc 2001; 54: 720-723

\section{Corresponding Author}

\section{S. Varadarajulu, M.D.}

Division of Gastroenterology-Hepatology University of Alabama at Birmingham Medical Center 410 LHRB, 1530 3rd Ave. S.

Birmingham

Alabama 35294

USA

Fax: $\quad+1-205-975-6381$

E-mail: svaradarajulu@yahoo.com 